\title{
El trastorno disártrico en Enfermedad de la motoneurona. Estudio descriptivo
}

\author{
Jesús García Tenorio \\ Facultad de Filosofia y letras \\ Universidad de Cádiz \\ Avda. Gómez Ulla, 1 \\ 11003 Cádiz \\ E-mail: jesgarten@hotmail.com
}

\section{EL TRASTORNO DISÁRTRICO EN ENFERMEDAD DE LA MOTO- NEURONA. ESTUDIO DESCRIP- TIVO}

RESUMEN: De entre los múltiples sintomas y trastornos que padecen las personas con Enfermedad de la Motoneurona, en lo referente a la comunicación oral, la disartria es el más generalizado. Cuando la zona bulbar se ve afectada, en el enfermo se produce un trastorno disártrico que afecta tanto a los órganos articulatorios activos como a la prosodia. Aunque el origen de dicha disartria es diferente en cada paciente, llegado a cierto punto en la evolución de esta enfermedad, los pacientes suelen presentar los mismos sintomas en los órganos relacionados con la comunicación oral: distonia de la musculatura respiratoria y orofacial, disartria espástica/flácida o mixta.

PALABRAS ClAVES: disartria; fonética; lingüística clínica; enfermedad de la motoneurona; ELA.

SUMARIO: 1. Introducción. 2. Presentación del estudio de casos. 3. Conclusiones.

\section{THE DYSARTHRIC DISORDER IN L'AFFECTION DE DYSARTHRIE MOTONEURON DISEASE. A DESCRIPTIVE STUDY DANS LA MALADIE DU CRIPTIVE}

ABSTRACT: People living with motoneuron disease suffer from multiple symptoms and disorders, with regard to oral communication, but dysarthria is the most common one. When the bulbar area is affected, in the patient it causes a dysarthric disorder which affects both active articulator organs and prosody. Although the origin of the dysarthric disorder is different in each patient, at some point in the evolution of the disease, patients show the same symptoms in the organs related to oral communication as dystonia in breathing muscles and orofacial musculature and spastic/flaccid dysarthria or a mix of both.

KEY WORDS: Dysarthria; Phonetics; Clinical Linguistics; Motoneuron disease; ALS.

SUMMARY: 1 . Introduction. 2. Conduct of the study. 3. Conclusion.
RÉSUMÉ: Parmi les plusieurs symptômes que souffrent les personnes avec la Maladie du motoneurone, en ce qui concerne la communication orale, la dysarthrie est le plus répandu. Lorsque la zone bulbaire est affectée, le malade est atteinte d'une affection de dysarthrie qui concerne tant les organes articulatoires actifs comme la zone d'articulation et la prosodie. Bien que l'origine de cette dysarthrie soit différente dans chaque patient, à un certain stade de cette maladie, il est fréquent que les patients présentent les mêmes symptômes aux organes impliqués dans la communication orale: dystonie de la musculature respiratoire et oro-faciale et dysarthrie spastique/flasque ou mixte.

MOTS CLÉS: $\begin{array}{r}\text { Dysarthrie; } \\ \text { Phonétique; }\end{array}$
$\begin{aligned} & \text { Linguistique } \\ & \text { clinique; }\end{aligned}$
motoneurone;
MLA.

SOMMAIRE: $1 . \quad$ Introduction. 2 clusions. Présentation de l'étude de cas. 3. Con- $\begin{array}{ll}\text { Fecha de Recepción } & 05 / 02 / 2019 \\ \text { Fecha de Revisión } & 19 / 03 / 2019 \\ \text { Fecha de Aceptación } & 10 / 05 / 2019 \\ \text { Fecha de Publicación } & 01 / 12 / 2020\end{array}$

DOI: http://doi.org/10.25267/Pragmalinguistica.2020.iextra2.10 


\title{
E1 trastorno disártrico en Enfermedad de la motoneurona. Es- tudio descriptivo
}

\author{
JESÚs GARCÍA TENORIO
}

\section{INTRODUCCIÓN}

Esta investigación, dentro del marco de una tesis doctoral y con el apoyo del Instituto de Investigación en Lingüística Aplicada (ILA) de la Universidad de Cádiz (UCA), la Fundación Española para el Fomento de la Investigación de la Esclerosis Lateral Amiotrófica (FUNDELA) y la Unidad Funcional de ELA del Hospital Universitario Puerta del Mar de Cádiz, pretende analizar el habla y la voz de las personas afectadas por la Enfermedad de la Motoneurona, para servir de ayuda a especialistas en logopedia, foniatría y audiología (Garcia Tenorio, 2016). A modo de introducción y para poder comprender mejor el trabajo llevado a cabo hasta el momento es necesario tener unas nociones terminológicas básicas.

En primer lugar, para poder hablar sobre trastornos disártricos, es conveniente saber, por un lado, que según el Diccionario Médico Online de la Clinica Universidad de Navarra, un trastorno consiste en la "presencia de un comportamiento o de un grupo de síntomas, identificables en la práctica clínica, que, en la mayoría de los casos, se acompañan de malestar o interfieren con la actividad del individuo" (En linea, s.v. trastorno) ${ }^{1}$, y por otro lado, poder delimitar las diferencias entre lo que son trastornos de la comunicación, del habla y del lenguaje. En sus Ensayos de Lingüistica General, Jakobson propone su Teoría de la comunicación, en la cual describió la comunicación (oral) como la relación existente entre emisor-receptor al intercambiar un mensaje (1984) y Saussure en su Curso de Lingüistica General marcó las diferencias entre el lenguaje, la facultad o capacidad comunicativa de los seres vivos; la lengua, el sistema de símbolos utilizado por una comunidad; y el habla, el uso individual de dicho sistema, como bien describe Amado Alonso en el prólogo de dicha obra (1945: 4-5).

En segundo lugar, según la Sociedad Española de Neurología (SEN) (2004: 2), "las enfermedades de la motoneurona son un grupo de enfermedades con afectación exclusiva o predominante de las neuronas motoras de la corteza cerebral, de los núcleos motores del troncoencéfalo y/o de las astas anteriores de la médula espinal". De entre todas las enfermedades que conforman este grupo de enfermedades podemos encontrar la Esclerosis Lateral Amiotrófica (ELA), la Atrofia Espinal Progresiva (AEP), la Parálisis Bulbar Progresiva (PBP) y la Esclerosis Lateral Primaria (ELP). No obstante, cuando se hace referencia a ella en singular, Enfermedad de la motoneurona, suele utilizarse como sinónimo de ELA o ALS (Amyotrophic Lateral

\footnotetext{
${ }^{1}$ Clínica Universidad de Navarra https://www.cun.es/diccionario-medico (Fecha de consulta
} 10/12/2018). 
Sclerosis) en su traducción al inglés (Sociedad Española de Neurología, 2004: 2-4) ${ }^{2}$.

Según la $A L S$ Association, la ELA es una enfermedad que puede tener uno de los siguientes tres orígenes. En primer lugar y con una tasa de aparición de casi en $90 \%$ de los casos, la ELA se manifiesta de manera esporádica; en segundo lugar y con una tasa de aparición del 5\%-10\% de los casos, la aparición de la enfermedad, es debida a causas genéticas. Este factor genético principalmente presente en el gen SOD1, es autosómico dominante y recesivo, es decir, que con que uno de los dos progenitores tenga la alteración genética podrán pasar ese gen defectuoso a sus hijos, los cuales podrian ser portadores; sin embargo, cuando los dos progenitores son portadores, la probabilidad de que el hijo resulte portador o portador afectado es del $75 \%$ de los casos. También se encuentran relacionados con la ELA genética otros genes como el TDP-43, FUS, alsina, senataxina y otros loci. En tercer lugar y con una tasa de aparición inferior al 5\% de los casos y de un probable origen tóxico, se encuentra la ELA Territorial, como es el caso de la ELA de la Isla de Guam o ELA Guamaniana (Mora Pardina, 2011: 10131014).

Las primeras manifestaciones de la enfermedad suelen ser de origen espinal, viéndose afectado principalmente el sistema periférico y la musculatura inherente a la capacidad respiratoria del enfermo, pero es la de afectación bulbar, inicial en el $25 \%$ de los casos, la representativa desde el punto de vista lingüístico, ya que afecta mayoritariamente a la musculatura orofacial.

Con el avance de la enfermedad comienzan a aparecer un sindrome bulbar con dificultad para la deglución tanto de sólidos como de líquidos y sialorrea, con lo cual se ve afectada la zona faríngea, atrofia de la lengua con fasciculaciones, reflujo nasal de líquidos y voz gangosa, por lo que se ve afectada la apertura y el cierre de la úvula y disartria, lo que implica debilidad y atrofia de la musculatura orofacial (González Díaz et al., 2003).

En tercer lugar, una disartria es una afectación del sistema nervioso, ya sea central o periférico, la cual produce alteraciones en el recorrido muscular, la fuerza, el tono, la velocidad y la precisión de los movimientos musculares implicados en la producción del lenguaje, es decir, de la musculatura implicada en la respiración, la fonación, la articulación y la resonancia del habla del enfermo (Melle Hernández, 2007: 13-14). No obstante, teniendo en cuenta factores perceptivos, acústicos y fisiológicos por medio de tecnologías de neuroimagen, los enfermos de ELA bulbar suelen verse afectados por disartria espástica, disartria flácida o ambas, según la evolución de la enfermedad.

2 Sociedad Española de Neurología www.sen.es/pdf/neuromuscular/motoneurona.pdf (Fecha de consulta 12/07/2018). 
Las personas afectadas por disartria espástica, generalmente tienen afectados los siguientes aspectos del lenguaje (Darley et al., 1969a y 1969b, apud Melle Hernández, 2007a: 19-22):

- Exceso o insuficiencia prosódica.

- Incompetencia articulatorio-resonadora.

- Estenosis fonatoria.

Las personas afectadas por disartria flácida, también conocida como parálisis pseudobulbar, pueden verse afectadas, según el lugar donde se produce la lesión (Darley et al., 1969a y 1969b, apud Melle Hernández, 2007a: 17-19) por:

- Insuficiencia fonatoria.

- Insuficiencia respiratoria.

- Insuficiencia fonatorio-prosódica.

Aunque existen más tipos de disartrias, nos hemos centrado en estas dos, pues son las más características de la Enfermedad de la Motoneurona. No obstante, todas ellas provocan en los enfermos tanto deficiencias estructurales con respecto a la respiración, la fonación, la articulación y la resonancia, como insuficiencias funcionales con respecto a la tasa de habla, la prosodia y la inteligibilidad (Melle Hernández, 2007b).

\section{Presentación del estudio de casos}

Este estudio tiene como finalidad analizar la evolución de la capacidad de habla de los enfermos diagnosticados de Esclerosis Lateral Amiotrófica dentro del marco de una investigación doctoral (García Tenorio, 2017). El estudio del lenguaje en esta dolencia puede ayudar tanto a la detección temprana de la existencia de la misma como al tratamiento paliativo realizado por profesionales de la logopedia, la foniatría y la audiología de manera que se mejore la calidad de vida de los pacientes. Las pruebas que hemos realizado de forma extraordinaria, es decir, que no se realizan normalmente a pacientes de la Unidad Funcional de ELA, han sido unas grabaciones de audio y vídeo, las cuales serán contrastadas para una mayor rigurosidad científica. Estas grabaciones serán analizadas para evaluar el grado de afectación lingüistica de los enfermos en un periodo de tiempo de un año.

A la lista de trastornos propios de la musculatura bulbar como son los casos de afectación afónica/disfónica, problemas disártricos/anártricos y las fasciculaciones, habría que añadir algunos trastornos secundarios a estos, como son la disfagia o la sialorrea. Inicialmente puede notarse en el enfermo de ELA bulbar, un cambio en el tono y la intensidad de la voz, así como un aumento de la nasalidad de los sonidos propiamente orales (Mascías Cadavid et al., 1999: 401-402). 
Previo análisis acústico de diez personas con Enfermedad de la motoneurona, se ha analizado in situ, la calidad del habla y los matices fonéticos que presentaban dichos enfermos, del mismo modo que se les ha hecho un cuestionario inicial para conocer su nivel de escolaridad, zona geográfica y otras patologias o trastornos que pudieran padecer.

A continuación, y utilizando una metodología inductiva, se ha analizado el habla de los pacientes para comprobar su nivel de inteligibilidad, ya que la calidad de dicha habla es esencial para el estudio de casos que estamos realizando, atendiendo a las características que presenta el habla de los afectados, tanto a nivel geográfico como a nivel patológico.

Tras ello, se ha elaborado una tabla de trastornos tanto respiratorios como fonatorios, articulatorios y prosódicos que puedan afectar a la praxis del habla. Tras un repaso de la bibliografia encontrada y bajo consulta a especialistas en logopedia y foniatría, se han descartado aquellos trastornos del habla y de la voz que no padecen este tipo de enfermos, pero hemos mantenido aquellos que sí se dan o sí suelen darse bajo esta sintomatología.

Una vez confeccionada, la mencionada tabla se ha usado posteriormente en consulta para marcar qué trastornos padece cada enfermo y poder hacer un estudio estadístico comparativo y evolutivo de cada paciente evaluado y tras concluir con las evaluaciones, con todas las muestras tomadas.

La realización de las grabaciones se ha dividido en dos partes. En primer lugar y con una duración aproximada de 5-7 minutos, se ha realizado una grabación a los pacientes hablando sobre un tema específico de su elección, en algunos casos, se ha intervenido en las grabaciones por petición o necesidad del paciente. En un segundo lugar, se ha realizado a los pacientes la Prueba de Evaluación Fonética (PEF) creada por África Herrera Fernández en la que se sigue la propuesta de Laura Bosh. Dicha prueba ha sido tomada y realizada bajo consentimiento del portal online ARASAAC ${ }^{3}$ y de la autora.

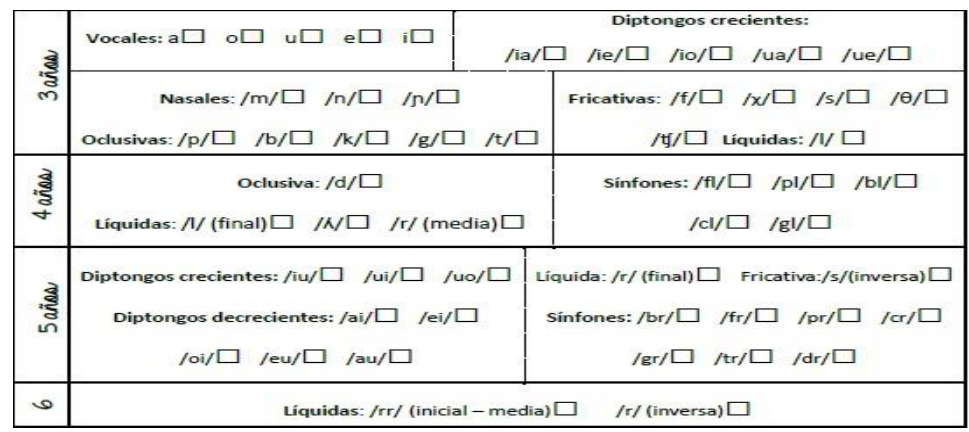

Imagen 1: Propuesta para la PEF por Herrera Fernández según propone Bosh (Herrera, 2017)

3 Centro Aragonés para la Comunicación Aumentativa y Alternativa http:/ /www.arasaac.org/ (Fecha de consulta 25/02/2018). 
Se ha utilizado esta prueba, no solo para anotar qué trastornos del habla y/o el lenguaje presentan los enfermos de ELA, sino también, para comprobar si hay un orden evolutivo decreciente en la pérdida de fonemas o de los sonidos conforme la afectación bulbar de la enfermedad avanza en el enfermo de ELA.

Dado que el presente proyecto consta de dos lineas de investigación bien diferenciadas pero complementarias, se plantean dos hipótesis principales.

Para la línea 1, centrada en los trastornos orales de la comunicación, la hipótesis de partida que refleja los objetivos planteados es:

Los diferentes trastornos de la comunicación oral en ELA se pueden categorizar mediante el estudio fonético-fonológico de la enfermedad, con el objeto de conseguir una metodología de evaluación específica y aplicable en la Unidades Funcionales de ELA.

Por otro lado, la hipótesis de partida propuesta para los estudios de casos longitudinales (línea 2) es:

Es posible describir un perfil lingüístico de la capacidad comunicativa de los pacientes con ELA, mediante diferentes estudios de casos longitudinales en los que se muestre la evolución de la capacidad comunicativa oral del paciente conforme va evolucionando la enfermedad.

Debido a la poca esperanza de vida que presentan los enfermos de ELA, a los pocos recursos que se les presta y a la falta de grupos de trabajo especializados en esta enfermedad, Unidades de ELA, este trabajo de investigación está basado en unos objetivos tanto generales como específicos con el fin de mejorar la calidad de vida del paciente, ofreciendo información sobre la etiología de la pérdida de las capacidades comunicativas orales desde el campo de la Lingüística a especialistas en Foniatría y Logopedia.

Los objetivos generales de esta investigación son:

- Categorizar los diferentes trastornos de la comunicación oral en ELA.

- Actualizar la información existente sobre los trastornos del lenguaje en ELA.

- Ofrecer más información sobre la etiología de la ELA desde el campo de la Lingüística Clínica a los especialistas y en especial a las Unidades Funcionales de ELA.

- Crear una guía lingüística para los especialistas en Foniatría y Logopedia.

Los objetivos específicos de esta investigación son:

- Identificar los trastornos orales de la comunicación especificos en pacientes con ELA.

- Clasificar los diferentes trastornos orales de la comunicación específicos en pacientes con ELA desde el campo de la Lingüística Clínica en la Fonética y la Fonología. 
- Analizar la etiología y las características específicas de los trastornos orales de la comunicación en pacientes con ELA desde el campo de la Lingüística Clínica en la Fonética y la Fonología.

- Establecer un protocolo lingüístico en la evaluación de la capacidad oral comunicativa del paciente con ELA desde el campo de la Lingüística Clínica en la Fonética y la Fonología.

- Establecer un perfil fonético y fonológico evolutivo de los pacientes con ELA mediante estudios de casos longitudinales.

Los pacientes han sido seleccionados de la Unidad Funcional de ELA del Hospital Universitario Puerta del Mar de Cádiz. Se considerarán candidatos todos aquellos hayan cumplido con los criterios de inclusión y con ninguno de los criterios de exclusión. No hay estudios previos sobre el objeto de la investigación que permitan calcular el tamaño de la muestra a estudio, por lo que, dado que las patologias de motoneurona son consideradas enfermedades raras y que no todos los afectados presentan afectación bulbar, se ha estimado como muestra representativa suficiente para obtener conclusiones significativas el análisis de 10 pacientes.

Los criterios de inclusión de los participantes han sido:

- Pacientes mayores de edad.

- Pacientes con diagnóstico de enfermedad de motoneurona que tengan afectación bulbar.

- Pacientes seguidos en la Unidad Funcional de ELA del Hospital Universitario Puerta del Mar de Cádiz.

- Pacientes que den su consentimiento informado por escrito para participar en el estudio.

Los criterios de exclusión de los participantes han sido:

- Pacientes que no tengan capacidad para hablar.

- Pacientes cuya situación física no les permita realizar las grabaciones.

- Pacientes que no den su consentimiento informado por escrito para participar en el estudio.

Para la realización de esta investigación, se han tenido en cuenta las variables estadísticas mostradas a continuación:

- Sexo.

- Edad.

- Zona geográfica procedente.

- Tiempo trascurrido desde la anamnesis.

- Variables articulatorias.

- Variables acústicas. 
Las actividades que se están realizando y se van a realizar durante la investigación son las que figuran a continuación:

- Análisis, recopilación y revisión heurística.

- Elaboración de una tabla sobre los trastornos orales en la comunicación.

- Análisis contrastivos de los trastornos fonético-fonológicos con respecto a la ELA.

- Creación de protocolo evaluador de trastornos fonético-fonológicos en pacientes con ELA.

- Utilización del protocolo evaluador con pacientes de la Unidad Funcional de ELA.

- Realización de los estudios de casos longitudinales de pacientes con ELA.

\section{Conclusiones}

Hasta el momento y tras una primera sesión de grabaciones con las 10 personas afectadas por la Enfermedad de la Motoneurona, se ha podido vislumbrar los siguientes datos:

De los diez enfermos de ELA que aceptaron en participar en la investigación, cinco presentaban, en el momento de la primera entrevista, afectación bulbar (Gráfico 1). En la mayoría de los casos el enfermo suele presentar sintomas de afectación espinal y tras la evolución de la enfermedad esta se solapa con la afectación bulbar.

Como podemos ver en el gráfico 1, solo un paciente mostraba inicialmente sintomas de afectación bulbar mientras que cinco presentaban solo sintomatología espinal, y cuatro pacientes presentaban tanto sintomatología espinal como bulbar, la cual suele ser la evolución típica de la esclerosis lateral amiotrófica.

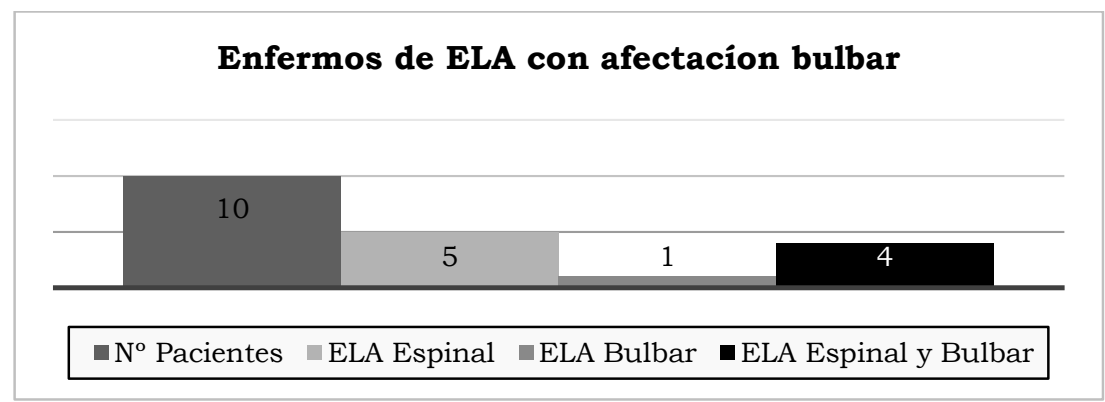

Gráfico 1: Enfermos de ELA con afectación bulbar.

E1 $100 \%$ de los casos estudiados con afectación bulbar (5 pacientes) ya presentaba problemas de disartria espástica en el momento de la primera grabación. Además, de todos los enfermos con afectación bulbar y disartria espástica, el 60\% de los casos (3 pacientes), también presentaba problemas 
de disartria flácida. No obstante, ninguno de los sujetos entrevistados presentaba sólo síntomas de disartria flácida.

Como resultado de la observación en consulta de la capacidad de habla de los pacientes, puede observarse la pérdida de la palatalización de los so-

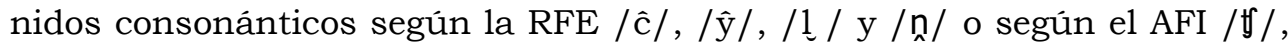
$/ \mathrm{ts} /, / \mathrm{d} 3 /, / 3 /, / \mathrm{K} /, / \mathrm{j} / \mathrm{y} / \mathrm{n} /$ o sus alófonos $[\mathrm{j}]$, como $\left[\mathrm{n}^{\mathrm{j}}\right]$ en la grafía del español -nch. También pueden observarse sonidos más nasalizados [ ], una tonalidad vocal forzada o estrangulada pero débil, en los que la movilidad mandibular y la apertura bucal oscila entre $10-25^{\circ}$ (Moya Molina et al., 2017: 228).

Se podría concluir, por tanto, de los datos obtenidos hasta la fecha que la disartria espástica aparece en primer lugar en el enfermo de ELA y luego, esta espasticidad se solapa con la disartria flácida conforme la enfermedad va avanzando, como podemos ver en el gráfico 2.

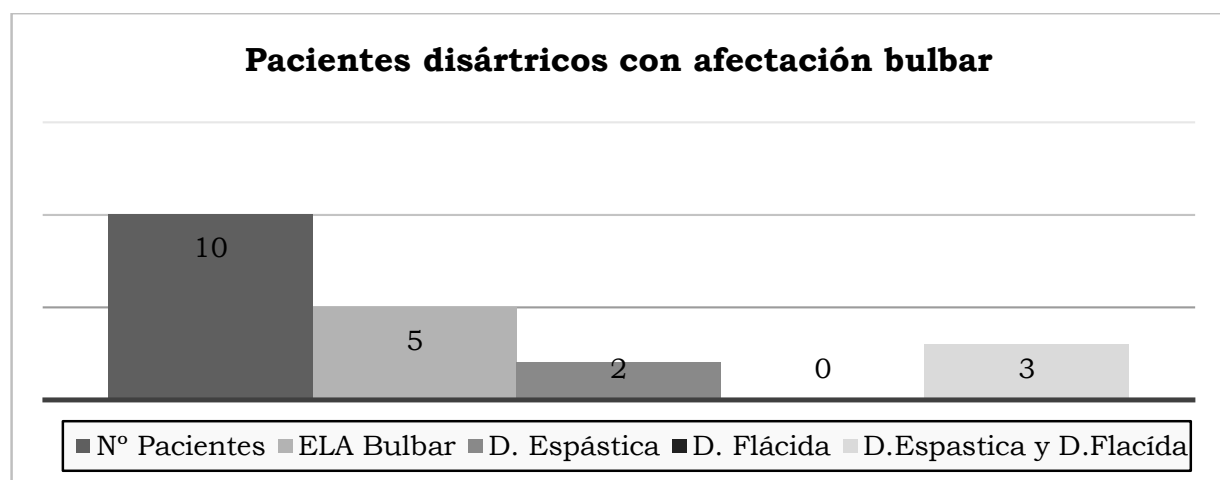

Gráfico 2: Pacientes disártricos con afectación bulbar.

Tras la primera sesión de grabaciones y entrevistas, los pacientes con disartria espástica o disartria espástica-flácida (mixta) presentaban un habla lenta, en algunos casos silabeada con una entonación inadecuada, una voz monótona y pérdida de la intensidad, así como frases cortas y capacidad discursiva reducida debido, mayoritariamente, a la insuficiencia respiratoria en aquellos casos más avanzados. La articulación vocálica y consonántica también se ha visto distorsionada, dándose bastantes casos de hipernasalidad mayoritariamente en los casos de disartria flácida. En estos últimos, también se ha percibido una voz más áspera, tensa y estrangulada; una voz mucho más grave que con respecto a los demás pacientes.

Aunque la investigación aún se encuentra en curso, los resultados obtenidos son bastante prometedores y se ajustan bastante a las hipótesis propuestas en la investigación, así como a la bibliografia consultada. Además, el análisis acústico de los pacientes promete ser bastante revelador con respecto a la etiología evolutiva de la enfermedad. 
Transcurrido un año desde las primeras grabaciones, se realizará una segunda grabación a los pacientes para de este modo poder contrastar ambas grabaciones y poder hacer un estudio evolutivo de la enfermedad en cada paciente. Por otro lado, una vez analizadas las grabaciones individuales de cada paciente, se contrastarán todos los datos obtenidos de todos los enfermos para de este modo intentar hallar patrones en la etiología y evolución de los trastornos del habla disártrica en los enfermos de ELA desde el punto de vista de la fonética articulatoria y acústica.

\section{REFERENCIAS}

CLÍNICA UNIVERSIDAD DE NAVARRA (2018): Diccionario médico. Disponible en: https://www.cun.es/diccionario-medico (Fecha de consulta: 03/01/2020).

DARLEY, F. L. et al. (1969a): "Clusters of deviant speech dimensions in the dysarthrias", Journal of Speech and Hearing research, 12, pp. 462-469.

DARLEY, F. L. et al. (1969b): "Differential diagnostic patterns of dysarthria", Journal of Speech and Hearing research, 12, p. 246.

GARCÍA TENORIO, J. (2016): "Esclerosis Lateral Amiotrófica y Lenguaje: Trastornos de la comunicación oral", Diéguez-Vide, F. (ed.): Temas de Lingüistica Clinica, Barcelona: Horsori, pp. 6773.

GARCÍA TENORIO, J. (2017): "La involución de la comunicación oral en pacientes con Esclerosis Lateral Amiotrófica. Cinco estudios de casos longitudinales", Fernández Gómiz, S.; Martínez Aguirre, R.; Núñez Bayo, Z. y Ureña Tormo, C. (eds.): Investigaciones actuales en Lingüistica Vol. VII: Planes de investigación de doctorado, Alcalá de Henares: Universidad de Alcalá, pp. 313-322.

GONZÁLEZ DİAZ, N. et al. (2003): "Esclerosis lateral amiotrófica. Monografia", Revista Mexicana de Medicina Física y Rehabilitación, 15, pp. 44-54.

HERRERA FERNÁNDEZ, A. (2017): Prueba de evaluación fonética
(PEF). Publicación electrónica http://www.arasaac.org/materiales.php?id_material $=1359$.

JAKOBSON, R. (1984): Ensayos de lingüística general, Barcelona: Ariel.

MASCÍAS CADAVID, J. et al. (1999): "Tratamiento de los problemas de comunicación", Mora Pardina, J. S. (ed.): Esclerosis Lateral Amiotrófica. Una enfermedad tratable, Madrid: Prous Science, pp. 401411.

MELLE HERNÁNDEZ, N. (2007a): Guia de intervención logopédica en disartria, Madrid: Sintesis.

MELLE HERNÁNDEZ, N. (2007b): "Intervención logopédica en la disartria", Revista de Logopedia, Foniatria y Audiología, 27 (4), pp. 187-197

MORA PARDINA, J. S. (2011): "Esclerosis Lateral Amiotrófica", Pascual Gómez, J. (ed.): Tratado de neurología, Madrid: LUZÁN 5, pp. 1013-1048.

MOYA MOLINA, M. Á. et al. (2017): "Los marcadores lingüisticos en la Disartria de pacientes con enfermedad de la motoneurona. Estudio descriptivo", Revista de Neurologia, 64 (5), pp. 227-234.

SAUSSURE, F. de $\left(1945,24^{\mathrm{a}}\right.$ edic.): Curso de Lingüistica General, Buenos Aires: Losada.

SOCIEDAD ESPAÑOLA DE NEUROLOGÍA (2004): Enfermedades de la motoneurona. Disponible en: www.sen.es/pdf/neuromuscular/motoneurona.pdf (Fecha de consulta: 10/02/2020). 